\title{
EFFECTS OF WASHING ON POSITIVE STAINING
}

\author{
KIYOTERU TOKUYASU
}

\author{
Department of Biology, University of California at San Diego, La Jolla, CA 92093-0322, USA
}

Received for publication December 9, 1991

\begin{abstract}
When sections embedded in epoxy resin were conventionally stained with lead citrate and washed very briefly, about $1 \mathrm{sec}$, a very high staining intensity was attained. However, the staining intensity was quickly reduced by washing $10 \mathrm{~min}$. When uranyl acetate-stained sections were washed for $1 \mathrm{sec}$, a much lower intensity was obtained. Nevertheless, a significant reduction in staining intensity occurred when washing was prolonged to $10 \mathrm{~min}$. These observations indicated that not only in staining of frozen sections or sections embedded in hydrophilic resins but also those embedded in more hydrophobic resins, the staining intensity is closely related to the degree of washing, particularly in lead-staining.
\end{abstract}

When ultrathin frozen sections were stained with uranyl acetate (UA) and then washed with water as commonly done to stain plastic-embedded sections, cellular structures were found to be much more weakly stained than in those in plastic embedded sections. To overcome this difficulty and also to protect frozen sections from damaging of surface tension at the time of drying, we eliminated the washing step and instead, embedded frozen sections in a thin layer of a mixture of a stain such as UA and a polymeric material such as methyl cellulose or polyvinyl alcohol at the final step of staining $(2,3)$. In other words, in this procedure which we called 'adsorption staining method', frozen sections were simultaneously stained and embedded in a protective layer at the time of drying.

When water miscible resins such as Lowicryl or LR White acrylic resin were used for embedding to carry out immunocytochemistry, cells were often fixed with formaldehyde and dehydrated without post-fixation with $\mathrm{OsO}_{4}$. Consequently, membranes were extracted during dehydration and recognized as linear vacancies in positively stained sections. Furthermore, when embedded in such resins, the staining intensity was found to be considerably lower than when embedded in more hydrophobic resins such as epoxy resins. We considered that hydration of the sections during washing caused removal of a major portion of UAstain in a manner similar to the case of staining frozen sections. In fact, when sections embedded in Lowicryl K4M were adsorption-stained with a mixture of UA and methl cellulose, the staining intensity was markedly increased (1). In this case, methyl cellulose in the staining mixture was believed to have served for uniform distribution of the stain over the resin-embedded sections.

These observations prompted us to study how washing affects the staining intensity in sections of commonly used, more hydrophobic resins such as epoxy resins. Here, we report that washing significantly affects the staining intensity of sections embedded in epoxy resin, in particular, in $\mathrm{Pb}$-staining.

\section{MATRIALS AND METHODS}

Small segments of intestine were dissected out from rats anaesthetized with barbital and after cutting to $0.5-1 \mathrm{~mm}$ size pieces, fixed in a mixture of $4 \%$ formaldehyde and $1 \%$ glutaraldehyde in $0.1 \mathrm{M}$

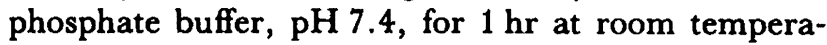
ture. The tissue pieces were then washed in phosphate buffer for $1 \mathrm{hr}$ to overnight and post-fixed with $2 \% \mathrm{OsO}_{4}$ in phosphate buffer for $1 \mathrm{hr}$ at room temperature. They were then washed with water (total 30$60 \mathrm{~min}$ ), dehydrated with ascending concentrations of acetone, processed through ascending concentration of Spurr's epoxy resin in acetone, and finally embedded in the resin.

Ultrathin sections of $70-80 \mathrm{~nm}$ thickness (silver color), cut with a Reichert UM-2 microtome, were stained with lead citrate $(\mathrm{Pb})(4)$ or uranyl acetate (UA) and then washed for $1 \mathrm{sec}$ or $10 \mathrm{~min}$ before drying, as described in detail in the Results.

To evaluate different staining intensities, all electron optical and photographic conditions were made constant as critically as possible. Original negatives 
were taken on film plates under the same conditions of exposure, at the same magnification $(\times 7,400)$ and at the same degree of underfocus $(5 \mu \mathrm{m})$. To compare a section washed for $1 \mathrm{sec}$ with that washed for $10 \mathrm{~min}$, plates of the two sections were put in th same rack and developed together, to eliminate possible photographic errors.

Nevertheless, in the electron microscope used in the present study (Philips EM-300), the brightness of a field was decided by the average electron density over the field, which caused the background electron density significantly greater in plates of more strongly stained sections than in those of more weakly stained ones. Therefore, the exposure time for printing was adjusted so that the background density of prints of different plates became approximately uniform.

\section{RESULTS AND DISCUSSION}

Sections were stained with $\mathrm{Pb}$ in an ordinary manner, that is, by floating the grids on $0.3 \% \mathrm{~Pb}$ for $3 \mathrm{~min}$. Subsequnet washing was carried out in two different ways. The shortest washing was achieved by touching the grid very briefly, approximately $1 \mathrm{sec}$, to the surface of a $1 \mathrm{~cm}$ water puddle on a Parafilm sheet. The grid was then picked up with a pair of tweezers and dried after removing remaining water with filter paper. More extensive washing was carried out by first floating the grids on each of two water puddles for $10 \mathrm{sec}$ and then leaving on the third puddle for $10 \mathrm{~min}$. The brief washing resulted in a very strong staining intensity (Fig. 1), which appeared to be even stronger than that resulting from the conventional double staining with $\mathrm{UA}$ and $\mathrm{Pb}$ (not shown). The staining intensity was, however, rapidly reduced by washing for $10 \mathrm{~min}$ (Fig. 2).

When sections were stained with UA for $10 \mathrm{~min}$ and washed very briefly in the same manner as done after $\mathrm{Pb}$-staining, the staining intensity was found to be considerably weaker than that resulting from the brief washing of $\mathrm{Pb}$-stained sections (compare Fig. 3 with Fig. 1). Nevertheless, various organelles were clearly delineated in such sections.
When sections were washed for $10 \mathrm{~min}$, ribosomes appeared to be similar in density to those washed for $1 \mathrm{sec}$ (compare arrowheads in Fig. 4 with those in Fig. 3). However, vacuoles that were visible in sections washed for $1 \mathrm{sec}$ (arrows in Fig. 3) were not readily recognizable in sections washed for $10 \mathrm{~min}$ (Fig. 4). Furthermore, membranes of mitochondria or endoplasmic reticulum that were clearly visible in sections washed for $1 \mathrm{sec}$ (Fig. 3) were not clearly recognizable in sections washed for $10 \mathrm{~min}$ (Fig. 4). These observations indicated that in UA-staining too, the staining intensity is significantly reduced by washing, albeit less drastically than in Pb-staining.

It is to be emphasized that Figs. 2-4 were printed in such a manner as presented here with the purpose of comparing them with Fig. 1. In fact, when Figs. 2 and 3 were printed more densely on papers of stronger contrast, the delineation of organelles was considerably improved (not shown). In other words, it was not that cell structures in Figs. 2 and 3 were impractically low in contrast but that the staining intensity of those in Fig. 1 was extremely high.

The above observations indicate that not only in staining of frozen sections or sections embedded in hydrophilic resins but also those embedded in more hydrophobic resins, the staining intensity is closely related to the degree of washing, particularly in leadstaining. The optimal conditions of washing for conventional staining procedures may need to be reevaluated by considering these results together with the possibility that short washing may tend to cause deposition of stain crystals upon drying of the sections.

\section{ACKNOWLEDGEMENT} 30282 .

This work was supported by NIH grant HL-

\section{REFERENCES}

1. Roth, J., Taatjes, D. J. and Tokuyasu, K. T.: Contrasting of Lowicryl K4M thin sections. Histochemistry 95; 123-136, 1990.

Micrographs presented here were printed on a medium contrast paper in such a manner that the background densities of Figs. 2-4 were approximately matched with that of Fig. 1. All are enlarged to the same magnification. Bar in Fig. 4, $0.5 \mu \mathrm{m}$.

Figs. 1, 2. Pb-staining for $3 \mathrm{~min}$ was followed by washing for $1 \mathrm{sec}$ (Fig. 1) or $10 \mathrm{~min}$ (Fig. 2). All organelles, including mitochondria $(\mathrm{m})$, endoplasmic reticulum (R), Golgi apparatus (G) and coated vesicles (arrows), are found to be much denser in Fig. 1 than in Fig. 2. In Fig. 1, a desmosome (D) and microtubules (arrowheads) are also clearly delineated.

Figs. 3, 4. UA-staining for $10 \mathrm{~min}$ was followed by washing for $1 \mathrm{sec}$ (Fig. 3) or $10 \mathrm{~min}$ (Fig. 4). Although ribosomes are recognized in Fig. 4 in approximately the same density as in Fig. 3, vesicles that are recognizable in Fig. 3 (arrows) are not clearly seen in Fig. 4. Membranes of other organelles (e.g., those of mitochondrial cristae (arrowheads) and endoplasmic reticulum (R) are also less distinct in Fig. 4 than in Fig. 3. 


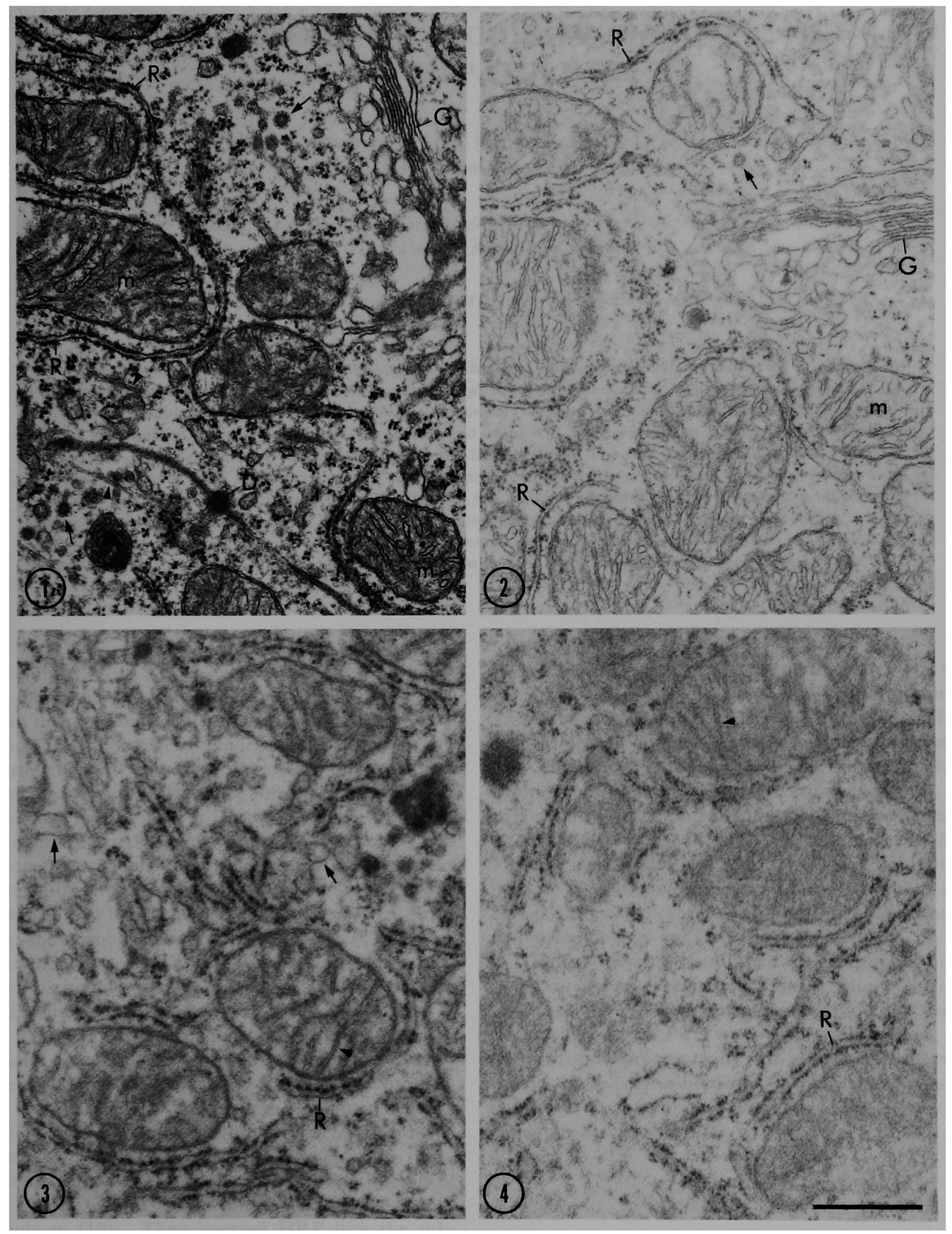


2. Tokuyasu, K. T.: Immunochemistry on ultrathin frozen sections. Histochem. J. 12; 381-403, 1980.

3. Tokuyasu, K. T.: Use of poly (vinylpyrrolidine) and poly (vinyl alcohol) for cryoultramicrotomy. Histochem. J. 21;
163-171, 1989.

4. Venable, J.M. and Coggeshall, R.: A simplified lead citrate stain for use in electron microscopy. J. Cell Biol. 25; 407-408, 1965. 